\section{P2-306 PLASMA HOMOCYSTEINE LEVELS ACCORDING TO METHYLENETETRAHYDROFOLATE REDUCTASE GENOTYPE AND SERUM FOLATE LEVELS IN A POPULATION-BASED STUDY IN SãO PAULO, BRAZIL}

doi:10.1136/jech.2011.142976k.39

V T Baltar, ${ }^{*}$ J Steluti, R S Bigio, G J F Gattás, R M Fisberg, D M L Marchioni. University of São Paulo, São Paulo, Brazil

Introduction Hyperhomocysteinemia is a risk factor of cardiovascular disease. Homocysteine remethylation requires vitamin $\mathrm{B}_{12}$, folate and methylenetetrahydrofolate reductase (MTHFR) enzyme. The common TT homozygosis of the C677T in the MTHFR gene is associated with reduced MTHFR activity. This study aims to assess the impact of serum levels of $B_{12}$ and folate on plasma homocysteine considering C677T polymorphism in a Brazilian sample.

Methods Serum vitamin $\mathrm{B}_{12}$, folate, and homocysteine of 259 participants from a population-based survey in São Paulo, Brazil were used. The genotype for C677T was done with an allele-specific polymerase chain reaction. A generalised linear model with gamma distribution and link identity was applied to model homocysteine according to sex, age, vitamin $\mathrm{B}_{12}$ as well as folate (cut-off at tercile $7.1 \mathrm{ng} / \mathrm{ml}$ ) and C677T polymorphism (non-TT and TT) interaction. Results Significant effects of males $(p<0.01)$ and age $(p<0.01)$ were found. An increase of $50 \mathrm{pg} / \mathrm{ml}$ in vitamin $B_{12}$ was associated with a reduction of $0.11 \mathrm{ng} / \mathrm{ml}$ in homocysteine levels $(p=0.01)$. Finally, an interaction between polymorphism and folate was found $(p<0.01)$, controlling all the covariates. A mean difference of $5.7 \mathrm{ng} / \mathrm{ml}$ of homocysteine levels was observed between below and above folate tercile among TT genotype $(\mathrm{p}<0.01)$ with a difference of only $1.1 \mathrm{ng} / \mathrm{ml}$ among non-TT $(p<0.01)$. Homocysteine levels among participants with above tercile of folate were similar between nonTT and TT ( $\mathrm{p}=0.57)$.

Conclusion Lower levels of folate are associated with higher levels of homocysteine, but in the presence of TT homozygote homocysteine is even higher.

\section{P2-307 LATENT MODEL FOR DNA METHYLATION, NUCLEOTIDE SYNTHESIS AND IMMUNE ACTIVATION FOR LUNG CANCER RISK}

doi:10.1136/jech.2011.142976k.40

${ }^{1} \mathrm{~V}$ T Baltar, ${ }^{2} \mathrm{~W}$ Xun, ${ }^{2} \mathrm{~S}-\mathrm{C}$ Chuang, ${ }^{3} \mathrm{P}$ Ferrari, ${ }^{3} \mathrm{M}$ Johansson, ${ }^{3} \mathrm{P}$ Brennan, ${ }^{2} \mathrm{P}$ Vineis, ${ }^{3}$ on behalf of the EPIC Working Group. ' University of São Paulo, São Paulo, Brazil; ${ }^{2}$ Imperial College London, London, UK; ${ }^{3}$ International Agency for Research on Cancer, Lyon, France

Introduction Lung cancer (LC) remains the leading cause of cancer mortality worldwide. In addition to tobacco exposure, low intake of specific micronutrient has been linked to LC. The diet is the main source of vitamins and amino acids involved in the one-carbon metabolism, which is considered key mechanism in maintaining DNA integrity, regulating gene expression, and may thus affect carcinogenesis. Two important branches of the one-carbon metabolism are implicated in cancer: DNA methylation (MET) and nucleotide synthesis (NS). In addition, immune activation (IA) is involved in the ageing process in normal healthy individuals and in a number of pathologies, including cancer.

Methods To investigate the three pathways and their relationships with LC, we applied structural equation models to relate three latent variables corresponding to each mechanism to LC status, controlling for independent effects of tobacco exposure (plasma cotinine). Each latent variable represents one of the mechanisms: MET (methionine, cobalamin, folate and serine), NS (folate, serine, vitamin $\mathrm{B}_{6}$, and Riboflavin) and IA (vitamin $\mathrm{B}_{6}$, Kynurenine/tryptophan ratio and Neopterin). The analysis was conducted using a data set from a nested case-control from the European Prospective Investigation into Cancer and Nutrition cohort.

Results We have found a direct and protective effect for MET $(p=0.011)$ and IA $(p=0.006)$, meanwhile NS presented only an indirect protective effect $(p=0.012)$.

Conclusion In conclusion, our results support the roles for MET and IA in LC aetiology, whereas the factor representing NS also showed some weak indirect associations. Tobacco remains the predominant predictive factor for LC.

\section{P2-308 THE CHARACTERISTICS OF PEOPLE WHO HAD BEEN SCREENED TO BE HYPERGLYCAEMIC BUT DID NOT VISIT CLINICS: A RETROSPECTIVE COHORT STUDY}

doi:10.1136/jech.2011.142976k.41

${ }^{1} \mathrm{Y}$ Tsujimura, ${ }^{*} \mathrm{~T}$ Nakayama, ${ }^{1} \mathrm{~T}$ Ishizaki, ${ }^{1} \mathrm{Y}$ Takahashi, ${ }^{1} \mathrm{~K}$ Miyazaki, ${ }^{2} \mathrm{~T}$ Satoh, ${ }^{3} \mathrm{~S}$ Ikeda, ${ }^{4} \mathrm{~S}$ Kimura. ${ }^{1}$ Department of Health Informatics, Kyoto University School of Public Health, Kyoto City, Japan, ${ }^{2}$ Kitasato Clinical Research Center, Kitasato University School of Medicine, Tokyo, Japan; ${ }^{3}$ Department of Pharmaceutical Sciences, School of Pharmacy, International University and Health Welfare, Tokyo, Japan; ${ }^{4}$ Japan Medical Data Center Co.,Ltd., Tokyo, Japan

Introduction Diabetes poses a significant threat to quality of life due to serious complications including cardiovascular diseases, and as such, many discussions have focused on screening for diabetes. In Japan, the special health examination ("Tokutei Kenshin") launched in 2008 for all people aged 40-74 includes screening for diabetes. However, some individuals do not make follow-up visits to see a doctor even if they are deemed to be hyperglycaemic at a health check-up.

Objectives To investigate proportions and characteristics of those deemed to be hyperglycaemic through screening but who did not make a follow-up visit.

Design A retrospective cohort study.

Setting and Participants The Japan Medical Data Center's database includes data from health insurance claims and health checkups for 109212 insured individuals.

Main outcome measures Prevalence of follow-up visits after health checkups.

Results We identified 4869 individuals deemed to be hyperglycaemic at health checkups (prevalence, 7.1\%). Of these, 2432 (49.9\%) did not make follow-up visits after health checkups (men $=49.1 \%$, women $=64.6 \%$ ). Elderly participants were more likely to follow-up after a health check-up where they were told to be hyperglycaemic (OR for NOT making follow-up clinical visit [95\% CI]: 10 years older, 0.70 [0.65 to 0.75 ] for men, 0.77 [0.59 to 1.01] for women).

Conclusions In the present study, almost half of individuals did not follow-up after a health check-up where they were deemed to be hyperglycaemic. Older individuals were more likely to follow-up than younger individuals. Participant characteristics should be taken into account as improvements are made to health check-up services.

\section{P2-309 IMPACT OF EXCESS WEIGHT ON THE RELATIONSHIP BETWEEN BLOOD PRESSURE AND CARDIOVASCULAR DISEASE: THE ASIA PACIFIC COHORT STUDIES COLLABORATION}

doi:10.1136/jech.2011.142976k.42

${ }^{1} \mathrm{R}$ Tsukinoki, ${ }^{2} Y$ Murakami, ${ }^{3} \mathrm{R}$ Huxley, ${ }^{4} \mathrm{~T}$ H Lam, ${ }^{5} \mathrm{X}$ Fang, ${ }^{6} \mathrm{I}$ Suh, ${ }^{7} \mathrm{~T}$ Ohkubo, ${ }^{8} \mathrm{H}$ Ueshima, ${ }^{1} \mathrm{M}$ Woodward. ${ }^{1}$ The George Institute for Global Health, University of Sydney, Sydney, Australia; ${ }^{2}$ Department of Medical Statistics, Shiga University of Medical Science, Otsu, Shiga, Japan; ${ }^{3}$ Division of Epidemiology, University of Minnesota, Minnesota, USA; ${ }^{4}$ Department of Community Medicine, University of Hong 